\title{
Fatty acid profile of cultured green abalone (Haliotis fulgens) exposed to lipid restriction and long-term starvation
}

\section{Perfil de ácidos grasos de organismos juveniles de abulón azul (Haliotis fulgens) sometidos a restricción de lípidos e inanición}

\author{
Eduardo Durazo ${ }^{1 *}$, María Teresa Viana² \\ ${ }^{1}$ Facultad de Ciencias Marinas, Universidad Autónoma de Baja California, AP 76, CP 22800, Ensenada, Baja \\ California, México \\ 2 Instituto de Investigaciones Oceanológicas, Universidad Autónoma de Baja California, AP 453, CP 22800, \\ Ensenada, Baja California, México \\ * Corresponding author: E-mail: edurazo@uabc.edu.mx
}

\begin{abstract}
The fatty acid composition of juvenile green abalone (Haliotis fulgens) exposed to lipid restriction and long-term starvation was studied. Juvenile organisms were acclimated during 26 days and then randomly separated into three treatment groups. One group was fed a restricted diet containing low lipid content $(0.14 \%)$, another was fed a rich diet containing the optimal lipid content (5.1\%), and the third was kept under starvation conditions. After 90 days, the abalone fed the restricted diet showed a significant increase in 18:1n-9 content and a decrease in n-3 and n-6 polyunsaturated fatty acids (PUFA), but the total lipid level remained similar to that observed before the treatment, suggesting lipogenesis. On the other hand, no changes in total lipid content and fatty acid profile were found during the 90 -day treatment using the rich lipid diet compared with the initial samples. Starved abalone showed that lipids did not constitute the main energy source and that the concentrations of long-chain PUFA did not change throughout the 90-day starvation period.
\end{abstract}

Key words: green abalone, formulated feed, dietary lipid level, starvation, fatty acid composition.

RESUMEN. Se estudió la composición de ácidos grasos de juveniles de abulón azul (Haliotis fulgens) sometidos a restricción de lípidos y a un periodo largo de inanición. Los organismos juveniles se aclimataron durante 26 días y posteriormente se separaron al azar en tres grupos de tratamiento. Un grupo se alimentó con una dieta baja en lípidos $(0.14 \%)$, otro se alimentó con una dieta rica en lípidos (5.1\%) y el tercer grupo se mantuvo en condiciones de inanición. Después de 90 días los abulones alimentados con la dieta baja en lípidos mostraron un incremento del contenido de 18:1n-9 y una disminución de los ácidos grasos poliinsaturados (PUFA) n-3 y n-6, pero el contenido de lípidos totales permaneció similar al observado antes del tratamiento, sugiriendo lipogénesis. Por otra parte, después de 90 días no se encontraron cambios en cuanto al contenido de lípidos totales y el perfil de ácidos grasos en el tratamiento con la dieta rica en lípidos en comparación con las muestras iniciales. Los abulones en inanición mostraron que los lípidos no constituyen la fuente principal de energía y que las concentraciones de PUFA de cadena larga no cambian a lo largo de un periodo de inanición de 90 días.

Palabras clave: abulón azul, alimento formulado, nivel de lípidos en la dieta, inanición, composición de ácidos grasos.

\section{INTRODUCTION}

Abalone species are distributed worldwide and they are found in temperate as well as in tropical waters. Due to overexploitation the natural populations of some commercial species, with high market value, are declining dramatically (Guest et al. 2008). Considerable research has thus been conducted on their culture and nutrition over the past 30 years with success.

The green abalone (Haliotis fulgens) is a herbivorous gastropod with high cultivation potential. The distribution of this species includes southern California and the west coast of the Baja California Peninsula, Mexico. There is growing interest in farming and marketing this highly prized product, especially in the USA and Chile. In addition, the unusual energy and lipid metabolism of $H$. fulgens has led to several studies (Floreto et al. 1996, Durazo-Beltrán et al. 2004, Viana et al.

\section{INTRODUCCIÓN}

Las especies de abulón tienen una distribución mundial y se encuentran en aguas tanto templadas como tropicales. Debido a su sobreexplotación, las poblaciones naturales de algunas especies de alto valor comercial están disminuyendo drásticamente (Guest et al. 2008). Por esta razón, durante los últimos 30 años se han realizado diversas investigaciones sobre su cultivo y nutrición con resultados exitosos.

El abulón azul (Haliotis fulgens) es un gasterópodo herbívoro con gran potencial acuicultural. Su distribución incluye el sur de California y la costa occidental de la península de Baja California, México. Existe un creciente interés en el cultivo y la comercialización de este producto muy apreciado, especialmente en los Estados Unidos y Chile. Además, el metabolismo energético y de lípidos inusual de $H$. fulgens ha propiciado muchos estudios (Floreto et al. 
2007, Hernández et al. 2013). Dietary lipids, in addition to being an energy source, are the source of essential fatty acids that are not synthesized by the organism and that are necessary for cellular metabolism and maintenance of the membrane structure (Corraze 2001). In most aquatic organisms, including abalone, the main function of both $n-3$ and $n-6$ polyunsaturated fatty acids (PUFA) appears to be structural, and their use as an energy source is considered to be limited (Floreto et al. 1996, Sargent et al. 2002). Some reports on the green abalone have shown that neutral lipids are metabolized to produce energy only when the other sources, like proteins and carbohydrates, are depleted. Proteins are preferentially used as an energy source to maintain the lipid reserves (Segawa 1993, Durazo-Beltrán et al. 2004). On the other hand, several investigations with different abalone species (Haliotis discus hannai, $H$. fulgens, $H$. laevigata, and H. rubra) have shown that the growth rate and fatty acid composition of the different tissues are affected by both the quality and the quantity of dietary lipids (Uki et al. 1986, Durazo-Beltrán et al. 2003a, Grubert et al. 2004). It has been suggested that $H$. fulgens is able to desaturate and elongate the fatty acids $18: 2 \mathrm{n}-6$ and $18: 3 \mathrm{n}-3$ to synthesize the longchain polyunsaturated fatty acids (LC-PUFA) 20:4n-6 (arachidonic acid) and 20:5n-3 (eicosapentanoic acid), respectively (Durazo-Beltrán 2003a, 2003b).

To our knowledge, no information is available on longterm starvation experiments on abalone, and it has been pointed out that more studies are needed on the lipid requirements of green abalone and their association with parameters such as growth rate, inanition, reproduction, and metabolism (Nelson et al. 2002, Viana et al. 2007). Thus, the main goal of the present work was to study the effect of the level of dietary lipids and of a long period of starvation on the fatty acid composition of muscle tissue of cultured juvenile H. fulgens.

\section{MATERIALS AND METHODS}

Juvenile $H$. fulgens $(298 \pm 30 \mathrm{mg} ; 28.0 \pm 0.2 \mathrm{~mm})$ were obtained from a commercial farm (BC Abalone, Ejido Eréndira, Baja California, Mexico). The organisms were acclimated for 26 days in a flow-through system consisting of 3.8-L plastic containers supplied with aerated and filtered seawater $\left(300 \mathrm{~mL} \mathrm{~min}^{-1}\right)$, and fed a standard formulated diet (table 1). Eight abalone were placed in each container and maintained under constant temperature $\left(20.0 \pm 1.2^{\circ} \mathrm{C}\right)$ and a photoperiod of $12 \mathrm{~h} \mathrm{light} / 12 \mathrm{~h}$ darkness. After the acclimation period, three treatment groups were randomly separated. One group was offered a rich lipid diet (RLD), another was offered a low lipid diet (LLD), and the third was kept under starvation conditions. All experiments were carried out in triplicate.

The experimental diets (table 1) were formulated to have similar concentrations of crude protein as previously described by Durazo-Beltrán et al. (2003a). LLD was
1996, Durazo-Beltrán et al. 2004, Viana et al. 2007, Hernández et al. 2013). Los lípidos dietéticos, además de ser una fuente de energía, son una fuente de ácidos grasos esenciales que no son sintetizados por el organismo y que son necesarios para el metabolismo celular y el mantenimiento de la estructura de la membrana (Corraze 2001). En la mayoría de los organismos acuáticos, incluyendo el abulón, la función principal de los ácidos grasos poliinsaturados (PUFA, por sus siglas en inglés) n-3 y n-6 parece ser estructural y su uso como fuente de energía se considera limitado (Floreto et al. 1996, Sargent et al. 2002). Algunos reportes del abulón azul han mostrado que los lípidos neutreos se metabolizan para producir energía sólo cuando se agotan las otras fuentes, como las proteínas y los carbohidratos. Las proteínas se usan preferentemente como fuente de energía para mantener las reservas de lípidos (Segawa 1993, Durazo-Beltrán et al. 2004). Por otro lado, varios estudios con diferentes especies de abulón (Haliotis discus hannai, $H$. fulgens, $H$. laevigata y H. rubra) han mostrado que la tasa de crecimiento y la composición de ácidos grasos en distintos tejidos resultan afectadas por tanto la calidad como la cantidad de lípidos en la dieta (Uki et al. 1986, Durazo-Beltrán et al. 2003a, Grubert et al. 2004). Se ha sugerido que $H$. fulgens es capaz de desaturar y elongar los ácidos grasos 18:2n-6 y 18:3n-3 para sintetizar los ácidos grasos poliinsaturados de cadena larga (LC-PUFA) 20:4n-6 (ácido araquidónico) y 20:5n-3 (ácido eicosapentaenoico), respectivamente (Durazo-Beltrán 2003a, 2003b).

Hasta donde sabemos, no hay información sobre experimentos de inanición a largo plazo para el abulón, y se ha comentado que es necesario realizar estudios adicionales de los requerimientos lipídicos del abulón azul y su asociación con parámetros como la tasa de crecimiento, inanición, reproducción y metabolismo (Nelson et al. 2002, Viana et al. 2007). Por lo tanto, el objetivo principal de este trabajo fue estudiar los efectos del nivel de lípidos dietéticos y de un periodo largo de inanición en la composición de ácidos grasos del tejido muscular de juveniles cultivados de $H$. fulgens.

\section{MATERIALES Y MÉTODOS}

Se obtuvieron especímenes juveniles de $H$. fulgens $(298 \pm 30 \mathrm{mg} ; 28.0 \pm 0.2 \mathrm{~mm})$ de una granja comercial (BC Abalone, Ejido Eréndira, Baja California, México). Los organismos se aclimataron durante 26 días en un sistema de contenedores de plástico de $3.8 \mathrm{~L}$ con flujo abierto de agua de mar aireada y filtrada $\left(300 \mathrm{~mL} \mathrm{~min}{ }^{-1}\right)$, y se alimentaron con una dieta estándar (tabla 1). Se colocaron ocho especímenes en cada contenedor y se mantuvieron bajo temperatura constante $\left(20.0 \pm 1.2^{\circ} \mathrm{C}\right)$ y un fotoperiodo de $12 \mathrm{~h} \mathrm{luz} / 12 \mathrm{~h}$ oscuridad. Después del periodo de aclimatación, se separaron tres grupos al azar. Un grupo se alimentó con una dieta rica en lípidos, otro recibió una dieta baja en lípidos y el tercero se mantuvo en condiciones de inanición. Todos los experimentos fueron realizados por triplicado. 
prepared to contain a very low amount of lipids, only $0.14 \%$ $(w / w)$, using ingredients defatted with hot ethanol and no lipid supplement, whereas RLD was formulated to satisfy the metabolic requirements of abalone and contained 5.10\% (w/w) of lipids (Durazo-Beltrán et al. 2003a). All ingredients were blended with $50 \%$ water until a completely homogeneous dough-like mixture was obtained. The diets were then rolled flat to a thickness of $2 \mathrm{~mm}$, and $10 \times 5 \mathrm{~mm}$ pieces were cut and stored in airtight plastic containers at $-25^{\circ} \mathrm{C}$ until use. Proximate analysis of each experimental diet was conducted according to standard methods (AOAC 1995). Three abalone were sampled from each replicate for total lipid and fatty acid analysis at the start of the experiment and another three were sampled at the end of the treatment on day 90. In the starvation treatment, muscle samples were also taken on days 50 and 70 .

Total lipid content in diets and muscle was determined by extraction using chloroform:methanol $(2: 1, \mathrm{v} / \mathrm{v})$ following the extraction method described by Folch et al. (1957). Analysis of fatty acid methyl esters (FAME) was performed according to Christie (1993) using a Hewlett Packard 6890II gas chromatograph equipped with a flame ionization detector and a capillary column (Omegawax 320, Supelco/SigmaAldrich; $30 \mathrm{~m} \times 0.32 \mathrm{~mm}$, film thickness $0.25 \mathrm{~mm}$ ). Fatty acids were identified by comparing their retention times with those of well-characterized FAME standards (37-Component FAME Mix, PUFA1, PUFA3, Supelco/Sigma-Aldrich). Each fatty acid concentration was estimated from the corresponding chromatogram area using an internal standard (19:0) and the Agilent ChemStation (version E.02.00.493) software package.

All data were subjected to one-way analysis of variance. Differences were considered statistically significant at $P<$ 0.05 . Means were compared after analysis of variance by Tukey range tests. All statistical analyses were carried out using Minitab v16.2 (Minitab Inc., State College, PA, USA). The results are reported as mean \pm standard error of the mean.

\section{RESULTS}

The two experimental diets (RLD and LLD) were formulated to have similar protein/energy ratios, but different lipid content (table 1). The levels of monounsaturated fatty acids (MFA), PUFA, and LC-PUFA in both RLD and LLD were lower than those found in a standard diet (table 2), whereas the level of saturated fatty acids (SFA) was higher in RLD. The level of 18:2n-6 in LLD was 24-fold lower than that in RLD, resulting in a higher $n-3 / n-6$ ratio in LLD $(0.30)$ than that observed in RLD (0.02), mainly due to the content of 18:2n-6 from corn oil in the lipid mixture.

The lipid profiles of the abalone muscles were determined before and after each treatment (table 3). There were significant differences in the fatty acid profiles after the experimental feeding and starvation periods. At the beginning of the
Las dietas experimentales (tabla 1) se formularon para contener concentraciones similares de proteína cruda, como previamente descrito por Durazo-Beltrán et al. (2003a). La dieta baja en lípidos se preparó para contener una cantidad muy baja de éstos, sólo $0.14 \%(\mathrm{p} / \mathrm{p})$, con ingredientes desengrasados con etanol caliente y sin suplemento lipídico, mientras que la dieta rica en lípidos se formuló para satisfacer los requerimientos metabólicos del abulón, con $5.10 \%(\mathrm{p} / \mathrm{p})$ de lípidos (Durazo-Beltrán et al. 2003a). Todos los ingredientes se mezclaron en una mezcladora con $50 \%$ de agua hasta obtener una pasta homogénea. El alimento se aplanó a un espesor de $2 \mathrm{~mm}$ y se cortaron piezas de $10 \times 5 \mathrm{~mm}$, las cuales fueron almacenadas en recipientes herméticos de plástico a $-25^{\circ} \mathrm{C}$ hasta su uso. El análisis proximal de cada dieta experimental se realizó de acuerdo con métodos estándar (AOAC 1995). Se analizó el contenido de lípidos totales y ácidos grasos de tres especímenes de cada réplica al inicio del experimento así como de otros tres al final del experimento (día 90). En el tratamiento de inanición, también se tomaron muestras musculares los días 50 y 70 .

El contenido de lípidos totales en las dietas y el músculo se determinó mediante extracción con cloroformo y metanol $(2: 1, \mathrm{v} / \mathrm{v})$ siguiendo el método de extracción descrito por Folch et al. (1957). Los ésteres metílicos de ácidos grasos (FAME) se analizaron según Christie (1993), en un cromatógrafo de gases Hewlett Packard 6890II equipado con un detector de ionización de llama y una columna capilar (Omegawax 320, Supelco/Sigma-Aldrich; $30 \mathrm{~m} \times 0.32 \mathrm{~mm}$, película de $0.25 \mathrm{~mm}$ de espesor). Los ácidos grasos se identificaron comparando sus tiempos de retención con los de estándares de FAME bien caracterizados (37-Component FAME Mix, PUFA1, PUFA3, Supelco/Sigma-Aldrich). Se estimó la concentración de cada ácido graso del área correspondiente del cromatograma con un estándar interno (19:0) y el programa Agilent ChemStation (versión E.02.00.493).

Todos los datos fueron sometidos a un análisis de varianza de una vía. Las diferencias fueron consideradas estadísticamente significativas si $P<0.05$. Las medias se compararon después de un análisis de varianza mediante pruebas de rangos de Tukey. Todos los análisis estadísticos se realizaron con Minitab v16.2 (Minitab Inc., State College, PA, EUA). Los resultados se presentan como media \pm error estándar de la media.

\section{REsultados}

La dieta rica en lípidos y la dieta baja en lípidos se formularon con una razón proteína/energía similar pero diferente contenido de lípidos (tabla 1). Los niveles de ácidos grasos monoinsaturados (MFA), PUFA y LC-PUFA en ambas dietas fueron menores que los de la dieta estándar (tabla 2), mientras que el nivel de ácidos grasos saturados (SFA) fue mayor en la dieta rica en lípidos. El nivel de 18:2n-6 en la dieta baja en lípidos fue 24 veces menor que en la dieta rica en lípidos, 
Table 1. Ingredients ( $\mathrm{g} / 100 \mathrm{~g}$ diet) and proximate composition (g/100 g dry weight) of the standard diet used during the acclimatization period and the two experimental diets fed the green abalone Haliotis fulgens.

Tabla 1. Ingredientes ( $\mathrm{g} / 100 \mathrm{~g}$ dieta) y composición proximal ( $\mathrm{g} / 100 \mathrm{~g}$ peso seco) de la dieta estándar usada durante el periodo de aclimatación y de las dos dietas experimentales ofrecidas al abulón azul Haliotis fulgens.

\begin{tabular}{|c|c|c|c|}
\hline Ingredients & $\begin{array}{c}\text { Standard } \\
\text { diet }\end{array}$ & $\begin{array}{c}\text { Low lipid } \\
\text { diet }\end{array}$ & $\begin{array}{c}\text { Rich lipid } \\
\text { diet }\end{array}$ \\
\hline Corn starch & 4.34 & 29.08 & 24.08 \\
\hline Fish protein concentrate $^{a}$ & & 18.00 & 18.00 \\
\hline Albumin chicken egg ${ }^{b}$ & & 18.00 & 18.00 \\
\hline Soybean flour defatted & & 10.00 & 10.00 \\
\hline Cellulose & & 8.00 & 8.00 \\
\hline Modified $\operatorname{starch}^{\mathrm{c}}$ & 10.00 & 5.00 & 5.00 \\
\hline Gelatine (50 blooms) & 6.00 & 5.00 & 5.00 \\
\hline Lipids $^{\mathrm{d}}$ & & & 5.00 \\
\hline Mineral mixture ${ }^{\mathrm{e}}$ & 2.00 & 4.00 & 4.00 \\
\hline Vitamin mixture $^{\mathrm{f}}$ & 1.30 & 1.50 & 1.50 \\
\hline Fish silage: liquid phase ${ }^{g}$ & 2.00 & 1.00 & 1.00 \\
\hline Stay- $C^{h}$ & 0.40 & 0.20 & 0.20 \\
\hline Choline chloride & 0.11 & 0.10 & 0.10 \\
\hline Sodium benzoate & 0.23 & 0.10 & 0.10 \\
\hline Butylated hydroxytoluene & 0.09 & 0.01 & 0.01 \\
\hline$\alpha$-tocopherol & & 0.01 & 0.01 \\
\hline Fish meal ${ }^{\mathrm{i}}$ & 35.00 & & \\
\hline Kelp meal $^{\mathrm{j}}$ & 15.00 & & \\
\hline Corn meal $^{\mathrm{k}}$ & 12.00 & & \\
\hline Soybean meal ${ }^{1}$ & 10.00 & & \\
\hline Methionine & 0.23 & & \\
\hline \multicolumn{4}{|l|}{ Proximate composition } \\
\hline Crude protein & 36.47 & 33.81 & 35.26 \\
\hline Total lipid & 7.09 & 0.14 & 5.10 \\
\hline Ash & 20.79 & 5.73 & 5.25 \\
\hline Gross energy (kJ/g) & 17.36 & 18.33 & 19.41 \\
\hline $\mathrm{CP} / \mathrm{GE}$ ratio $(\mathrm{mg} / \mathrm{kJ})^{\mathrm{m}}$ & 21.01 & 18.45 & 18.17 \\
\hline
\end{tabular}

a $97 \%$ protein. ${ }^{b}$ Freeze-dried egg white. ${ }^{c}$ Modified corn starch (Clearjel). ${ }^{\mathrm{d}} 1.5 \%$ corn oil $+3.5 \%$ tripalmitin. ${ }^{\mathrm{e}}$ ICN salt mixture \#5 Briggs. ${ }^{\mathrm{f}}$ ICN vitamin diet fortification. ${ }^{\mathrm{g}}$ Acid fish silage from tuna viscera. ${ }^{\mathrm{h}}$ Ascorbyl polyphosphate (Roche). ${ }^{\mathrm{i}} 64 \%$ protein, $10 \%$ lipid. ${ }^{\mathrm{j}}$ Made from Macrocystis pyrifera. ${ }^{\mathrm{k}} 8 \%$ protein, $3.9 \%$ lipid.

${ }^{1} 39 \%$ protein, $21 \%$ lipids. ${ }^{\mathrm{m}}$ Crude protein/gross energy.

experiment, the most abundant fatty acids in muscle tissue were 16:0, 16:2n-6, 18:0, 18:1n-9, 20:5n-3, and 22:5n-3. After 90 days, the organisms fed LLD showed a higher amount of 18:1n-9 and a decrease in n-3 and n-6 LC-PUFA and the $n-3 / n-6$ ratio, while the total lipid level was similar
Table 2. Fatty acid and total lipid contents ( $\mathrm{mg} / \mathrm{g}$ dry weight) of the standard and experimental diets.

Tabla 2. Contenido de ácidos grasos y lípidos totales $(\mathrm{mg} / \mathrm{g}$ peso seco) de la dieta estándar y las dietas experimentales.

\begin{tabular}{|c|c|c|c|}
\hline Fatty acid & Standard diet & Low lipid diet & Rich lipid diet \\
\hline $14: 0$ & 2.15 & nd & 0.31 \\
\hline $16: 0$ & 14.57 & 0.36 & 33.92 \\
\hline $17: 0$ & 0.39 & nd & 0.06 \\
\hline 18:0 & 4.78 & 0.11 & 1.87 \\
\hline $20: 0$ & 0.31 & nd & 0.08 \\
\hline $22: 0$ & 0.09 & nd & 0.08 \\
\hline$\Sigma \mathrm{SFA}^{\mathrm{a}}$ & 22.29 & 0.47 & 36.32 \\
\hline $16: \ln -7$ & 2.35 & 0.05 & nd \\
\hline $18: \ln -9$ & 12.35 & 0.20 & 4.13 \\
\hline $18: 1 n-7$ & 2.54 & 0.02 & 0.20 \\
\hline $20: \ln -9$ & 1.29 & nd & 0.04 \\
\hline $22: 1 n-9$ & 0.40 & nd & 0.05 \\
\hline$\Sigma \mathrm{MFA}^{\mathrm{b}}$ & 18.93 & 0.27 & 4.42 \\
\hline $16: 3 n-6$ & 0.15 & 0.06 & 0.07 \\
\hline $18: 2 n-6$ & 13.14 & 0.34 & 8.11 \\
\hline $18: 2 n-4$ & 0.08 & nd & 0.07 \\
\hline $18: 3 n-3$ & 1.76 & 0.02 & 0.13 \\
\hline$\Sigma$ PUFA $^{\mathrm{c}}$ & 15.13 & 0.45 & 8.38 \\
\hline $20: 4 n-6$ & 0.52 & 0.03 & nd \\
\hline $20: 5 n-3$ & 3.12 & 0.03 & nd \\
\hline $22: 5 n-3$ & 0.55 & nd & nd \\
\hline $22: 6 n-3$ & 4.58 & 0.08 & 0.07 \\
\hline$\Sigma$ LC-PUFA $^{\mathrm{d}}$ & 8.77 & 0.14 & 0.07 \\
\hline$\sum n-3$ & 10.01 & 0.13 & 0.20 \\
\hline$\sum \mathrm{n}-6$ & 13.81 & 0.43 & 8.18 \\
\hline Ratio n-3/n-6 & 0.72 & 0.30 & 0.02 \\
\hline
\end{tabular}

nd: not detected. ${ }^{a}$ SFA: saturated fatty acids. ${ }^{b}$ MFA: monounsaturated fatty acids. ${ }^{\mathrm{c}}$ PUFA: polyunsaturated fatty acids, $<\mathrm{C}_{20} \geq 2$ double bonds $+\geq \mathrm{C}_{20} \leq 2$ double bonds. ${ }^{\mathrm{d}}$ LC-PUFA: long-chain polyunsaturated fatty acids, $\geq \mathrm{C}_{20} \geq 3$ double bonds.

por lo que la razón n-3/n-6 fue mayor en la primera $(0.30)$ que en la segunda (0.02), principalmente debido al contenido de 18:2n-6 del aceite de maíz en la mezcla de lípidos.

Los perfiles lipídicos del músculo de abulón se determinaron antes y después de cada tratamiento (tabla 3 ). Se observaron diferencias significativas en los perfiles de ácidos grasos después de los periodos de alimentación e inanición. $\mathrm{Al}$ inicio del experimento, los ácidos grasos más abundantes en el tejido muscular fueron 16:0, 16:2n-6, 18:0, 18:1n-9, $20: 5 n-3$ y $22: 5 n-3$. Después de 90 días, los organismos alimentados con la dieta baja en lípidos mostraron una mayor cantidad de 18:1n-9 y una disminución de los LC-PUFA n-3 y n-6 y de la razón n-3/n-6, mientras que el nivel de lípidos 
Table 3. Fatty acid and total lipid contents (mg/g dry weight) in muscle of juvenile Haliotis fulgens before any treatment, after being fed the experimental diets (90 days), and during starvation (mean \pm standard error, $n=3$ ).

Tabla 3. Contenido de ácidos grasos y lípidos totales ( $\mathrm{mg} / \mathrm{g}$ peso seco) en el músculo de organismos juveniles de Haliotis fulgens antes de cualquier tratamiento, después de recibir las dietas experimentales (90 días) y en condiciones de inanición (media \pm error estándar, $n=3$ ).

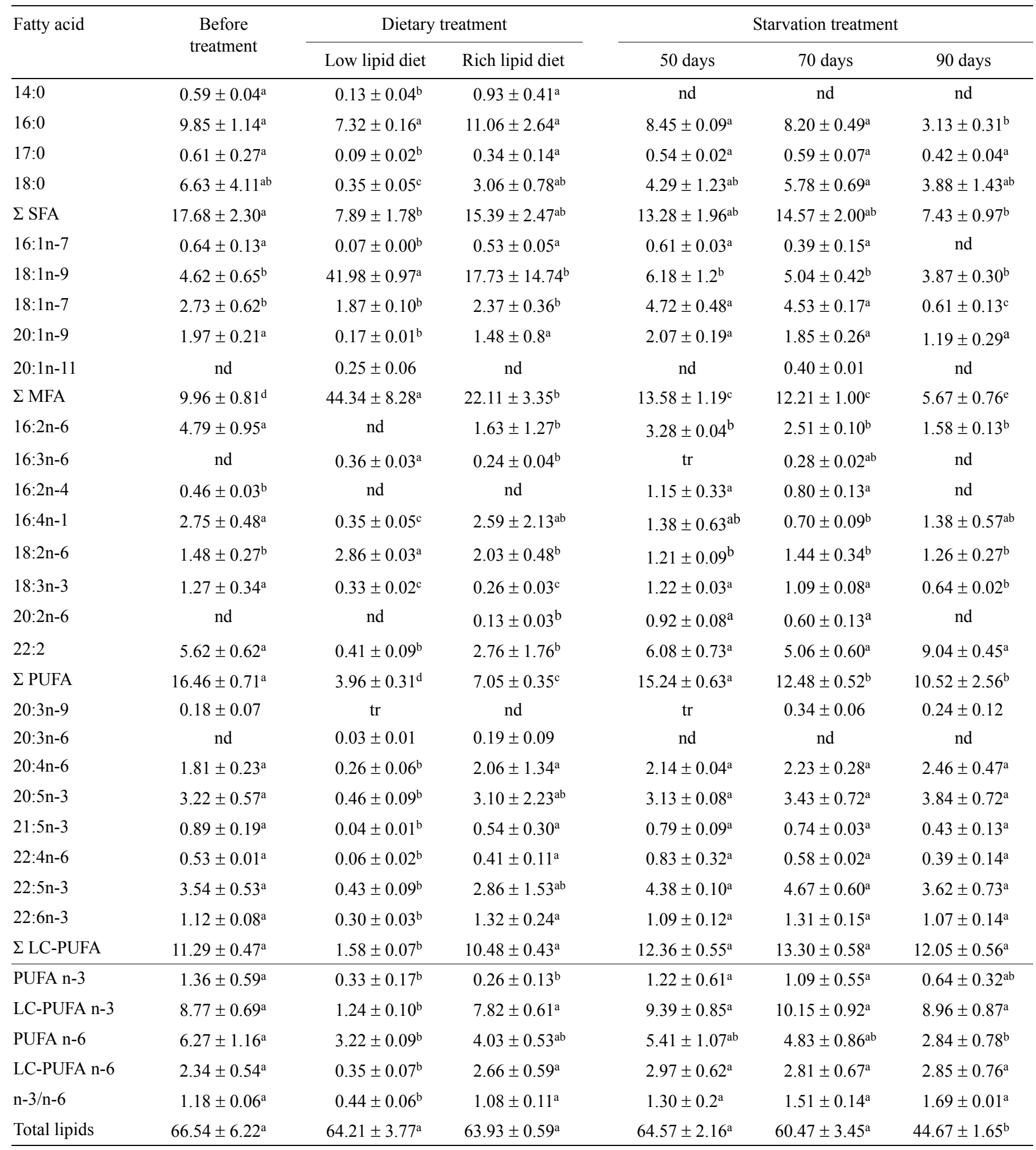

nd: not detected; tr: trace $(<0.01 \mathrm{mg} / \mathrm{g})$.

Means in the same row with different superscript are significantly different $(P<0.05)$. 
$(P>0.05)$ to that observed before the experiment. Abalone fed RLD showed similar values of SFA, 18:1n-9, 18:1n-7, $18: 2 n-6,20: 1 n-9,20: 2 n-6,20: 3 n-6,22: 4 n-6,22: 6 n-3$, and total lipids $(P>0.05)$ to those observed in abalone fed the standard diet until starting the treatment. No changes $(P>$ 0.05 ) in the fatty acid profiles and total lipid content of abalone were observed after 50 and 70 days of starvation. Nevertheless, abalone starved for 90 days showed significantly lower SFA, MFA, and total lipid contents $(P<0.05)$.

\section{Discussion}

The fatty acid profiles reflected the lipid source used for the experimental diets. LLD was designed to contain the lowest possible amount of lipids. It is not possible for a diet to be lipid-free because it is difficult to eliminate all the fat present in the diet components (Mai et al. 1995). Nevertheless, the total lipid level was much lower $(0.14 \% \mathrm{w} / \mathrm{w})$ than that contained in $\operatorname{RLD}(5.1 \% \mathrm{w} / \mathrm{w})$ or the recommended amounts.

Before treatment, the most abundant fatty acids of the muscle tissue were 16:0, 16:2n-6, 18:0, 18:1n-9, 20:5n-3, and $22: 5 n-3$, with a balanced n-3/n-6 ratio (1.18), in agreement with a previous report (Durazo-Beltrán et al. 2003a). This profile is originated by the dietary lipids from fish and vegetal sources, since abalone tend to accumulate n-3 LC-PUFA as 20:5n-3 and 22:5n-3 (Uki et al. 1986). At the end of the LLD treatment, the muscle tissue of abalone showed an increase in the amount of 18:1n-9 and a decrease in the n-3 and n-6 LC-PUFA contents, while the total lipid level remained unchanged when compared with the content before the treatment. The absence or presence of small amounts of n-3 and n-6 LC-PUFA in the diet and the occurrence of high levels of 18:1n-9 in the tissue could be an indication of essential fatty acid deficiency, and suggest negligible $\Delta 6$-desaturase activity which is needed to synthetize $18: 2 n-9$ and 20:2n-9 (Sargent et al. 1995, Ibeas et al. 1996). Consistently, Atlantic salmon fed a diet containing insufficient amounts of $\mathrm{n}-3$ and n-6 PUFA for four months showed that nearly $50 \%$ of the fatty acids in liver triacylglycerols corresponded to 18:1n-9 (Ruyter et al. 2000).

The total lipid content and fatty acid profile of abalone fed LLD suggest possible lipogenesis from carbohydrate or protein (Durazo-Beltrán et al. 2003a), which tends to reduce the LC-PUFA content and has been associated with tissue growth under fatty acid deficiency. Abalone fed RLD showed similar values of SFA, 18:1n-9, 18:1n-7, 18:2n-6, 20:1n-9, $20: 2 n-6,20: 3 n-6,22: 4 n-6,22: 6 n-3$, and total lipids to those recorded after the acclimatization period during which a standard diet was used (i.e., before treatment), but the levels of n-3 and 20:4n-6 LC-PUFA were lower. Starvation or restricted feeding is not unusual to marine invertebrates when food is scarce or unavailable over a long period of time, and the main response is a reduction in the metabolic rate to totales fue similar $(P>0.05)$ al observado antes del experimento. Los organismos alimentados con la dieta rica en lípidos mostraron valores de SFA, 18:1n-9, 18:1n-7, 18:2n-6, 20:1n-9, 20:2n-6, 20:3n-6, 22:4n-6, 22:6n-3 y lípidos totales similares $(P>0.05)$ a los de los organismos alimentados con la dieta estándar hasta el inicio del tratamiento. En el experimento de inanición, no se observaron cambios $(P>0.05)$ en los perfiles de ácidos grasos y el contenido de lípidos totales en los días 50 y 70; sin embargo, después de 90 días de inanición, los abulones presentaron contenidos significativamente menores $(P<0.05)$ de SFA, MFA y lípidos totales.

\section{DISCUSIÓN}

Los perfiles de ácidos grasos reflejaron las fuentes alimenticias de lípidos. La dieta baja en lípidos fue formulada para contener la menor cantidad posible de éstos. No es posible obtener una dieta totalmente libre de lípidos ya que resulta difícil eliminar toda la grasa de los componentes alimenticios (Mai et al. 1995). No obstante, el nivel de lípidos totales fue mucho menor $(0.14 \% \mathrm{p} / \mathrm{p})$ que el contenido en la dieta rica en lípidos $(5.1 \% \mathrm{p} / \mathrm{p})$ o que las cantidades recomendadas.

Al inicio del experimento, los ácidos grasos más abundantes en el tejido muscular fueron 16:0, 16:2n-6, 18:0, $18: 1 n-9,20: 5 n-3$ y $22: 5 n-3$, con una razón n-3/n-6 balanceada (1.18), lo cual coincide con un reporte previo (DurazoBeltrán et al. 2003a). Este perfil se debe a los lípidos de la dieta de fuentes vegetales y de pescado, ya que los abulones tienden a acumular LC-PUFA n-3 como 20:5n-3 y 22:5n-3 (Uki et al. 1986). Al final del tratamiento con la dieta baja en lípidos, el tejido muscular mostró un incremento del contenido de 18:1n-9 y una disminución de las concentraciones de LC-PUFA n-3 y n-6, mientras que el nivel de lípidos totales no cambió con respecto al observado antes del tratamiento. La ausencia o presencia de pequeñas cantidades de LC-PUFA n-3 y n-6 en la dieta y la presencia de niveles altos de 18:1n-9 en el tejido pueden ser indicios de una deficiencia de ácidos grasos esenciales y sugieren que fue despreciable la actividad de la enzima $\Delta 6$-desaturasa que se necesita para sintetizar 18:2n-9 y 20:2n-9 (Sargent et al. 1995, Ibeas et al. 1996). El salmón del Atlántico alimentado con una dieta formulada con cantidades insuficientes de los PUFA n-3 y n-6 durante cuatro meses, consistentemente mostró que casi el $50 \%$ de los ácidos grasos en los triacilgliceroles hepáticos correspondían a 18:1n-9 (Ruyter et al. 2000).

El contenido de lípidos totales y el perfil de ácidos grasos de los abulones alimentados con la dieta baja en lípidos sugieren la posible existencia de lipogénesis a partir de carbohidratos o proteínas (Durazo-Beltrán et al. 2003a), lo cual tiende a reducir el contenido de LC-PUFA y ha sido asociado con el crecimiento de tejido bajo deficiencia de ácidos grasos. Los abulones ofrecidos la dieta rica en lípidos presentaron valores de SFA, 18:1n-9, 18:1n-7, 18:2n-6, 20:1n-9, 
conserve energy (Hochachka and Somero 1984). In addition to the general reduction in total body lipids, starvation induces highly variable changes in muscle lipid content (McCue 2010). Our results show that the total lipid content and fatty acid profiles of abalone starved for 50 and 70 days remain similar to those fed the standard diet before the treatment. This observation suggests that during the first 70 days of starvation, the muscle lipids are not used as an energy source. A similar pattern has been reported for starved eel (Boëtius and Boëtius 1985) and shrimp (Sánchez-Paz et al. 2006). Previous studies on green abalone showed that lipid accumulation combined with weight loss indicates that during starvation, carbohydrate and protein, rather than lipids, are used as the principal source of energy (Viana et al. 2007), a condition that may be associated with a low oxidative metabolism (Segawa 1993) of adipose tissue. After 70 days of starvation, however, the total lipid level began to decrease and declined approximately $26 \%$ within the next 20 days (90 days starvation). The initial levels of LC-PUFA remained essentially unchanged throughout the 90-day starvation period. In contrast, after 90 days of starvation, the total lipid, SFA, MFA, and PUFA contents all decreased. LC-PUFA, the main lipid constituents of cell membranes because they are a major component of phospholipids, appear to be preferentially conserved in order to maintain the structural integrity of membranes and physiological needs (Sargent et al. 1995, Zabelinskii et al. 1999). The tendency to conserve n-3 LCPUFA has been reported in starved fish and abalone where SFA and MFA are preferentially mobilized, whereas the levels of LC-PUFA like 20:5n-3, 22:5n-3, and 22:6n-3 remain almost constant (Navarro and Gutiérrez 1995, Durazo-Beltrán et al. 2004). Although lipids in the green abalone seem to be primarily used for growth and gonad maturation (Ottaviani et al. 2011), during long-term starvation lipids can serve as an energy source.

In conclusion, our results show the high metabolic flexibility of abalone and their capacity to adapt to different situations, such as restricted feeding or starvation, where LCPUFA supply is insufficient and lipogenesis may occur in order to meet the most important physiological requirements.

\section{ACKNOWLEDGMENTS}

This project was financed by Universidad Autónoma de Baja California (internal project 0365). We thank the commercial farm $\mathrm{BC}$ Abalone for their kind donation of the abalone used in our experiment.

\section{REFERENCES}

AOAC. 1995. Association of Official Analytical Chemists. Official Methods of Analysis of AOAC International Vol. 1. AOAC International, Arlington, VA., $684 \mathrm{pp}$.

Boëtius I, Boëtius J. 1985. Lipid and protein content in Anguilla anguilla during growth and starvation. Dana 4: 1-17. 20:2n-6, 20:3n-6, 22:4n-6, 22:6n-3 y lípidos totales similares a los registrados al finalizar el periodo de aclimatización durante el cual fueron alimentados con una dieta estándar, pero los niveles de LC-PUFA n-3 y 20:4n-6 fueron menores. Para los invertebrados marinos, una alimentación restringida o inanición no es inusual cuando hay nula o poca disponibilidad de alimentos durante un periodo largo de tiempo y su principal respuesta es una reducción de la tasa metabólica para conservar energía (Hochachka y Somero 1984). Además de una reducción general de lípidos corporales, la inanición induce cambios muy variables en el contenido lipídico del músculo (McCue 2010). Nuestros resultados muestran que el contenido de lípidos totales y el perfil de ácidos grasos de los abulones sometidos a inanición durante 50 y 70 días fueron similares a los de los organismos ofrecidos una dieta estándar antes de iniciar el tratamiento. Esto sugiere que durante los primeros 70 días de inanición, los lípidos musculares no son usados como una fuente de energía. Se ha documentado un patrón similar para anguilas (Boëtius y Boëtius 1985) y camarones (Sánchez-Paz et al. 2006) sometidos a inanición. Estudios previos del abulón azul encontraron que una acumulación de lípidos aunado a una pérdida de peso indica que, en condiciones de inanición, se usan carbohidratos y proteínas más que lípidos como la principal fuente de energía (Viana et al. 2007); esto puede estar relacionado con un bajo metabolismo oxidativo del tejido adiposo (Segawa 1993). Después de 70 días de inanición, sin embargo, el nivel de lípidos totales comenzó a decrecer, disminuyendo $25 \%$ en los siguientes 20 días (90 días de inanición). Los niveles iniciales de LC-PUFA no cambiaron a lo largo del periodo de inanición de 90 días. En contraste, después de 90 días de inanición, los niveles de lípidos totales, SFA, MFA y PUFA sí decrecieron. Los LC-PUFA son los principales constituyentes lipídicos de las membranas celulares por ser un componente importante de los fosfolípidos y aparentemente son conservados preferentemente para mantener la integridad estructural de las membranas y las necesidades fisiológicas (Sargent et al. 1995, Zabelinskii et al. 1999). Esta tendencia de conservar LC-PUFA n-3 ha sido observada en peces y abulones sometidos a inanición, donde los SFA y MFA se mobilizan preferencialmente, mientras que los niveles de LC-PUFA como 20:5n-3, 22:5n-3 y 22:6n-3 permanecen casi constantes (Navarro y Gutiérrez 1995, Durazo-Beltrán et al. 2004). Aunque los lípidos en el abulón azul se usan principalmente para crecimiento y maduración gonádica (Ottaviani et al. 2011), durante periodos largos de inanición pueden ser usados como una fuente de energía.

En conclusión, nuestros resultados muestran la gran flexibilidad metabólica del abulón y su capacidad para adaptarse a diversas situaciones, como alimentación restringida e inanición, en donde la disponibilidad de LC-PUFA es insuficiente y lipogénesis puede suceder para satisfacer las necesidades fisiológicas más importantes. 
Christie WW. 1993. Preparation of ester derivatives of fatty acids for chromatographic analysis. In: Christie WW (ed.), Advances in Lipid Methodology. Vol. 2. Oily Press, Dundee, pp. 69-111.

Corraze G. 2001. Lipid nutrition. In: Guillaume J, Kaushik S, Bergot $\mathrm{P}$, Metailler R (eds.), Nutrition and Feeding of Fish and Crustaceans. Springer, Chichester, UK, pp. 111-130.

Durazo-Beltrán E, D’Abramo LR, Toro-Vásquez JF, VásquezPeláez C, Viana MT. 2003a. Effects of triacylglycerols in formulated diets on growth and fatty acid composition in tissue of green abalone. Aquaculture 224: 257-270. http://dx.doi.org/10.1016/S0044-8486(03)00223-0

Durazo-Beltrán E, Toro-Vázquez JF, Vásquez-Peláez C, Viana MT. 2003b. Effect of the seaweed Macrocystis pyrifera and a formulated diet on growth and fatty acid composition in the green abalone, Haliotis fulgens, under commercial culture conditions. Cienc. Mar. 29: 645-654.

Durazo-Beltrán E, Viana MT, D’Abramo LR, Del Toro-Vázquez JF. 2004. Effects of starvation and dietary lipid on the lipid and fatty acid composition of muscle tissue of juvenile green abalone (Haliotis fulgens). Aquaculture 238: 329-341. http://dx.doi.org/10.1016/j.aquaculture.2004.03.025

Floreto EA, Teshima S., Koshio S. 1996. The effects of seaweed diets on the lipid and fatty acids of the Japanese disc abalone Haliotis discus hannai. Fish. Sci. 62: 582-588.

Folch J, Lees M, Stanley S. 1957. A simple method for the isolation and purification of total lipids from animal tissues. J. Biol. Chem. 226: 497-509.

Grubert MA, Dunstan GA, Ritar AJ. 2004. Lipid and fatty acid composition of pre- and post-spawning blacklip (Haliotis rubra) and greenlip (Haliotis laevigata) abalone conditioned at two temperatures on a formulated feed. Aquaculture 242: 297-311. http://dx.doi.org/10.1016/j.aquaculture.2004.08.033

Guest MA, Nichols PD, Frusher SD, Hirst AJ. 2008. Evidence of abalone (Haliotis rubra) diet from combined fatty acid and stable isotope analyses. Mar. Biol. 153: 579-588. http://dx.doi.org/10.1007/s00227-007-0831-9

Hernández J, Matus de la Parra A, Lastra M, Viana MT. 2013. Effect of lipid composition of diets and environmental temperature on the performance and fatty acid composition of juvenile European abalone (Haliotis tuberculata L. 1758). Aquaculture 412-413: 34-40. http://dx.doi.org/10.1016/j.aquaculture.2013.07.005

Hochachka PW, Somero GN. 1984. Biochemical Adaptation. Princeton Univ. Press, Princeton, 537 pp.

Ibeas C, Cejas J, Gómez T, Jerez S, Lorenzo A. 1996. Influence of dietary n-3 highly unsaturated fatty acids levels on juvenile gilthead seabream (Sparus aurata) growth and tissue fatty acid composition. Aquaculture 142: 221-235.

Mai K, Mercer JO, Donlon J. 1995. Comparative studies on the nutrition of two species of abalone, Haliotis tuberculata L. and Haliotis discus hannai Ino. III. Response of abalone to various levels of dietary lipid. Aquaculture 134: 65-80.

McCue MD 2010. Starvation physiology: Reviewing the different strategies animals use to survive a common challenge. Comp. Biochem. Physiol. 156: 1-18.

http://dx.doi.org/10.1016/j.cbpa.2010.01.002

\section{Agradecimientos}

Este trabajo fue financiado por la Universidad Autónoma de Baja California (proyecto interno 0365). Agradecemos a la granja comercial $\mathrm{BC}$ Abalone su donación de los abulones usados en el experimento.

Traducido al español por Christine Harris.

Navarro I, Gutiérrez J. 1995. Fasting and starvation. In: Hochachka PW, Mommsen TP (eds.), Biochemistry and Molecular Biology of Fishes. Vol. 4. Elsevier Science, Amsterdan, pp. 393-434.

Nelson MM, Leighton DL, Phleger CF, Nichols PD. 2002. Comparison of growth and lipid composition in the green abalone, Haliotis fulgens, provided specific macroalgal diets. Comp. Biochem. Physiol. 131: 695-712.

Ottaviani E, Malagoli D, Franceschi C. 2011. The evolution of the adipose tissue: A neglected enigma. Gen. Comp. Endocrinol. 174: $1-4$. http://dx.doi.org/10.1016/j.ygcen.2011.06.018

Ruyter B, Røsjø C, Einen O, Thomassen MS. 2000. Essential fatty acids in Atlantic salmon: Time course of changes in fatty acid composition of liver, blood and carcass induced by a diet deficient in n-3 and n-6 fatty acids. Aquacult. Nutr. 6: 109-117.

Sánchez-Paz A, García-Carreño F, Muhlia-Almazán A, PeregrinoUriarte AB, Hernández-López J, Yepiz-Plascencia G. 2006. Usage of energy reserves in crustaceans during starvation: Status and future directions. Insect Biochem. Mol. Biol. 36: 241-249. http://dx.doi.org/10.1016/j.ibmb.2006.01.002

Sargent JR, Bell JG, Henderson RJ, Tocher DR. 1995. Requirement criteria for essential fatty acids. J. Appl. Ichthyol. 11: 183-198.

Sargent JR, Tocher DR, Bell JG. 2002. The lipids. In: Halver JE, Hardy RW (eds.), Fish Nutrition. Academic Press, San Diego, CA, pp. 181-257.

Segawa S. 1993. Oxygen consumption and ammonia excretion by the abalone Sulcus diversicolor aquatilis in starved conditions. Bull. Jpn. Soc. Sci. Fish. 57: 2001-2006.

Uki N, Sugiura M, Watanabe T. 1986. Requirement of essential fatty acids in the abalone Haliotis discus hannai. Bull. Jpn. Soc. Sci. Fish. 52: 1013-1023.

Viana MT, D’Abramo LR, González MA, García-Suárez JV, Shimada A, Vásquez-Peláez C. 2007. Energy and nutrient utilization of juvenile green abalone (Haliotis fulgens) during starvation. Aquaculture 264: 323-329.

http://dx.doi.org/10.1016/j.aquaculture.2007.01.004

Zabelinskii SA, Chevotareva MA, Kostkin VB, Krivchenko AI. 1999. Phospholipids and their fatty acids in mitochondria, synaptosomes and myelin from the liver and brain of trout and rat: A new view on the role of fatty acids in membranes. Comp. Biochem. Physiol. 124B: 187-193.

Received February 2013, received in revised form July 2013, accepted October 2013. 